\title{
Real-Time Image Processing by Distributed Arithmetic Implementation of Two- Dimensional Digital Filters
}

\author{
JEFF JAGGERNAUTH, ALEXANDER C. P. LOUI, AND \\ ANASTASIOS N. VENETSANOPOULOS, SENIOR MEMBER, IEEE
}

\begin{abstract}
This paper describes the implementation of two-dimensional recursive digital filters for real-time image processing. The filter structure is described by distributed arithmetic and the operations involved are memory fetches and additions. An error analysis of the twodimensional filter structure described by distributed arithmetic is outlined and the expression for the mean-squared error is derived. This approach is compared to an equivalent conventional filter structure using multipliers. It is demonstrated that the distributed arithmetic implementation off ers a significant reduction in cost as well as power consumption for real-time image processing. In addition, the hardware design of a second-order two-dimensional distributed filter is presented and its performance is examined. This filter is constructed using standard integrated circuits and memories and is capable of filtering images of sizes up to $256 \times 256$ pixels in real time.
\end{abstract}

\section{INTRODUCTION}

$\mathrm{R}$ ECENTLY there has been increasing interest in the use of two-dimensional (2-D) filters for processing sampled 2-D data. For example, the geophysics industry used 2-D filters for processing seismic records, gravity, and magnetic data. 2-D filters have found applications in many areas including those of image enhancement, restoration of linearly degraded images and biomedical picture processing.

There are three common methods of 2-D filter implementation: direct convolution, frequency domain techniques through the use of the fast Fourier transform (FFT), and realization through recursive implementation. The recursive technique is the most efficient in terms of memory requirements and speed of implementation for a large convolution area [1]. Recursive filters have also been designed to satisfy both magnitude and phase characteristics. These filters were realized as basic second-order sections or as a cascade of second-order sections [2].

Peled and Liu [3] outlined an approach to the implementation of one-dimensional recursive digital filter structures described by distributed arithmetic. This realization requires storing the finite number of possible outcomes of an arithmetic operation, as well as using them to obtain the next output sample through repeated additions and

Manuscript received March 22, 1982; revised May 8, 1985.

The authors are with the Department of Electrical Engineering, University of Toronto, Toronto, Ont., Canada M5S 1 A4. shifting operations. Taylor [4] has shown that high-order high-speed FIR filters can be designed using the distributed arithmetic structure in the multiplicative form.

In this paper, an extension of the distributed arithmetic approach to the realization of 2-D recursive filters is investigated. Specifically, an implementation scheme for a second-order section is presented. Subsequent sections include a detailed description of the different aspects of distributed arithmetic implementation of 2-D filters. They are as follows. Section II introduces the theory of distributed arithmetic as applied to 2-D filters. An implementation is presented for a second-order section. Section III examines the effects of using finite word-length arithmetic and the mean-squared error at the output of the filter described by distributed arithmetic is derived. In Section IV, hardware considerations for real-time digital image processing are examined and comparisons are made between the new and the conventional multiplier implementation. In addition, the tradeoffs between data throughput rate and cost are investigated. Finally in Section V, a hardware implementation of the distributed arithmetic 2-D IIR filter is presented. The sequence of operations is outlined for filtering a general $M \times N$ image and the performance of the hardware is examined.

\section{Distributed ARithmetic Implementation}

More recently, FIR filters became very popular primarily because of their ease in design and implementation. This, however, has not been the case for recursive filters. Their use has been limited [5] due to the difficulties encountered at earlier attempts in designing stable filters. Even more recently, new techniques [1], [6] made possible the design of stable filters, as well as approximate desired magnitude and phase characteristics.

A 2-D recursive digital filter is described by the linear difference equation

$$
y_{m, n}=\sum_{(k, l) \in R_{a}} a_{k, l} x_{m-k, n-l}-\sum_{\substack{(i, j) \in R_{b} \\(i+j) \neq 0}} b_{i, j} y_{m-i, m-j}
$$

where $x$ and $y$ are the input and output arrays, respectively, and $R_{a}$ and $R_{b} \in I_{s}^{2}$ where $I_{s}$ is the set of integers. The case 


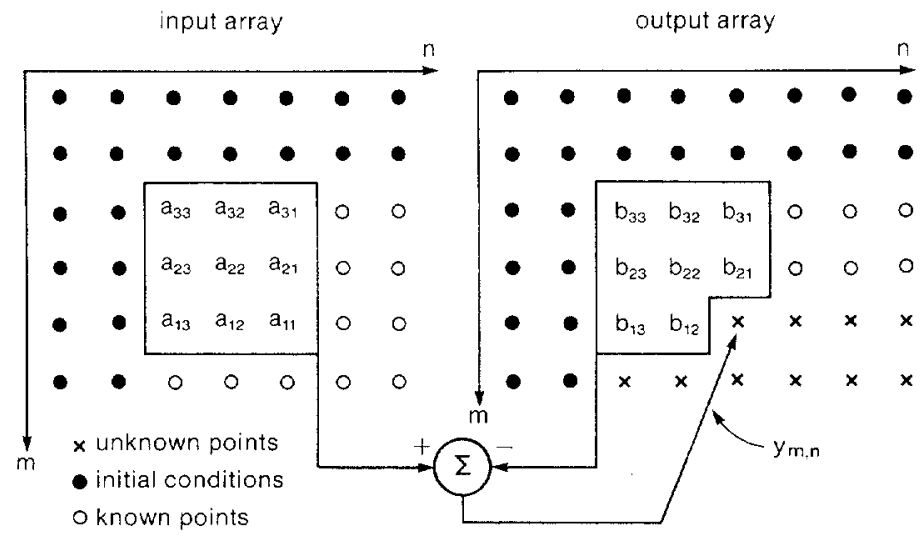

(a)

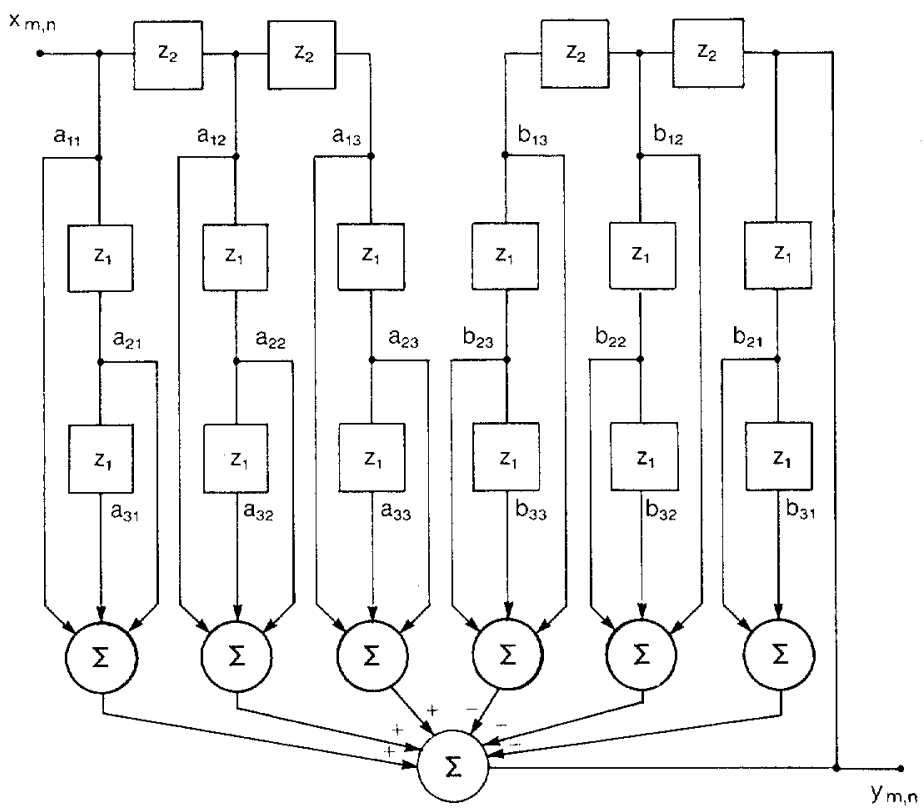

(b)

Fig. 1. (a) Input and output masks for a second-order filter. (b) Block diagram representation of a direct filtering process.

of $R_{a}$ and $R_{b}$ defined as

$$
\begin{array}{ll}
R_{a}=\{(k, l) \mid 0 \leq k \leq K 1, & 0 \leq l \leq L 1\} \\
R_{b}=\{(i, j) \mid 0 \leq i \leq I 1, & 0 \leq j \leq J 1\}
\end{array}
$$

indicates that the impulse response of the IIR filter is spread over only the upper quadrant of the right half plane in the spatial domain. This is referred to as a quarter plane impulse response. The filter is said to be causal [7] since its impulse response $h_{m, n}$ is zero for $M$ or $N$ less than zero. For a specific case, the sets $R_{a}$ and $R_{b}$, such that $K 1=L 1$ $=I 1=J 1=K$, the filter is referred to as of order $K$. In this paper, filters of order $K=2$ are considered. Those higher order filters designed as a cascade of second-order sections can be realized in a similar fashion. ${ }^{1}$

Fig. 1(a) shows an example of the filtering with a second-order section. In this particular case, the input mask $a_{k, l}$ is superimposed on the input image and the addition

'In general, a 2-D filter cannot be realized as a cascade; the cascade form is a special case. of the product between the mask and the underlying samples gives a 2-D convolution. The output mask $b_{i, j}$ is similar to the input except for the missing element in correspondence to the output sample to be computed. A direct realization of (1) is shown in Fig. 1(b). The $z_{1}$ and $z_{2}$ terms correspond to row and column delays, respectively. For each output sample, 17 multiplications, 15 additions, and one subtraction are required.

The general difference equation of (1) can be rewritten as

$$
y_{m, n}=\sum_{k=0}^{2} \sum_{l=0}^{2} a_{k, l} x_{m-k, n-l}-\sum_{\substack{i \\ i+j \neq 0}}^{2} \sum_{j}^{2} b_{i, j} y_{m-i, n-j}
$$

for a second-order filter. Assuming all signals to be bounded by \pm 1 and defining the input signal and output signal in two's complement code, $B$ bits of accuracy including the sign bit,

$$
x_{m-k, n-l}=\sum_{s=1}^{B-1} x_{m-k, n-l}^{s} 2^{-s}-x_{m-k, n-l}^{0}
$$

and

$$
y_{m-i, n-j}=\sum_{s=1}^{B-1} y_{m-i, n-j}^{s} 2^{-s}-y_{m-i, n-j}^{0}
$$

where $x_{m-k, n-l}^{s}$ and $y_{m-i, n-j}^{s}$ are binary variables.

Thus, (3) can be written as

$$
\begin{aligned}
y_{m, n}= & \sum_{k=0}^{2} \sum_{l=0}^{2} a_{k, l}\left(\sum_{s=1}^{B-1} x_{m-k, n-l}^{s} 2^{-s}-x_{m-k, n-l}^{0}\right) \\
& -\sum_{\substack{i=0 \\
i+j \neq 0}}^{2} \sum_{j=0}^{2} b_{i, j}\left(\sum_{s=1}^{B-1} y_{m-i, n-j}^{s} 2^{-s}-y_{m-i, n-j}^{0}\right) .
\end{aligned}
$$

Rearranging the summations in (5) yields

$$
\begin{aligned}
y_{m, n}= & \sum_{s=1}^{B-1}\left(\sum_{k=0}^{2} \sum_{l=0}^{2}\left(a_{k, l} x_{m-k, n-l}^{s}\right) 2^{-s}\right) \\
& -\sum_{k=0}^{2} \sum_{l=0}^{2}\left(a_{k, l} x_{m-k, n-l}^{0}\right) \\
& -\sum_{s=1}^{B-1}\left(\sum_{i=0}^{2} \sum_{j=0}^{2}\left(b_{i, j} y_{m-i, n-j}^{s}\right) 2^{-s}\right) \\
& +\sum_{\substack{i+j \neq 0 \\
i+j \neq 0}}^{2} \sum_{j=0}^{2}\left(b_{i, j} y_{m-i, n-j}^{0}\right) .
\end{aligned}
$$

Defining two functions

$$
\begin{aligned}
& F_{1}^{s}\left(x_{m, n}^{s}, x_{m, n-1}^{s}, x_{m, n-2}^{s}, \cdots, x_{m-2, n-2}^{s}\right) \\
& =a_{00} x_{m, n}^{s}+a_{01} x_{m, n-1}^{s} \\
& \quad+a_{02} x_{m, n-2}^{s}, \cdots, a_{22} x_{m-2, n-2}^{s}
\end{aligned}
$$

and

$$
\begin{aligned}
& F_{2}^{s}\left(y_{m, n-1}^{s}, y_{m, n-2}^{s}, \cdots, y_{m-2, n-2}^{s}\right) \\
& \quad=b_{01} y_{m, n-1}^{s}+b_{02} y_{m, n-2}^{s}+\cdots+b_{22} y_{m-2, n-2}^{s}
\end{aligned}
$$




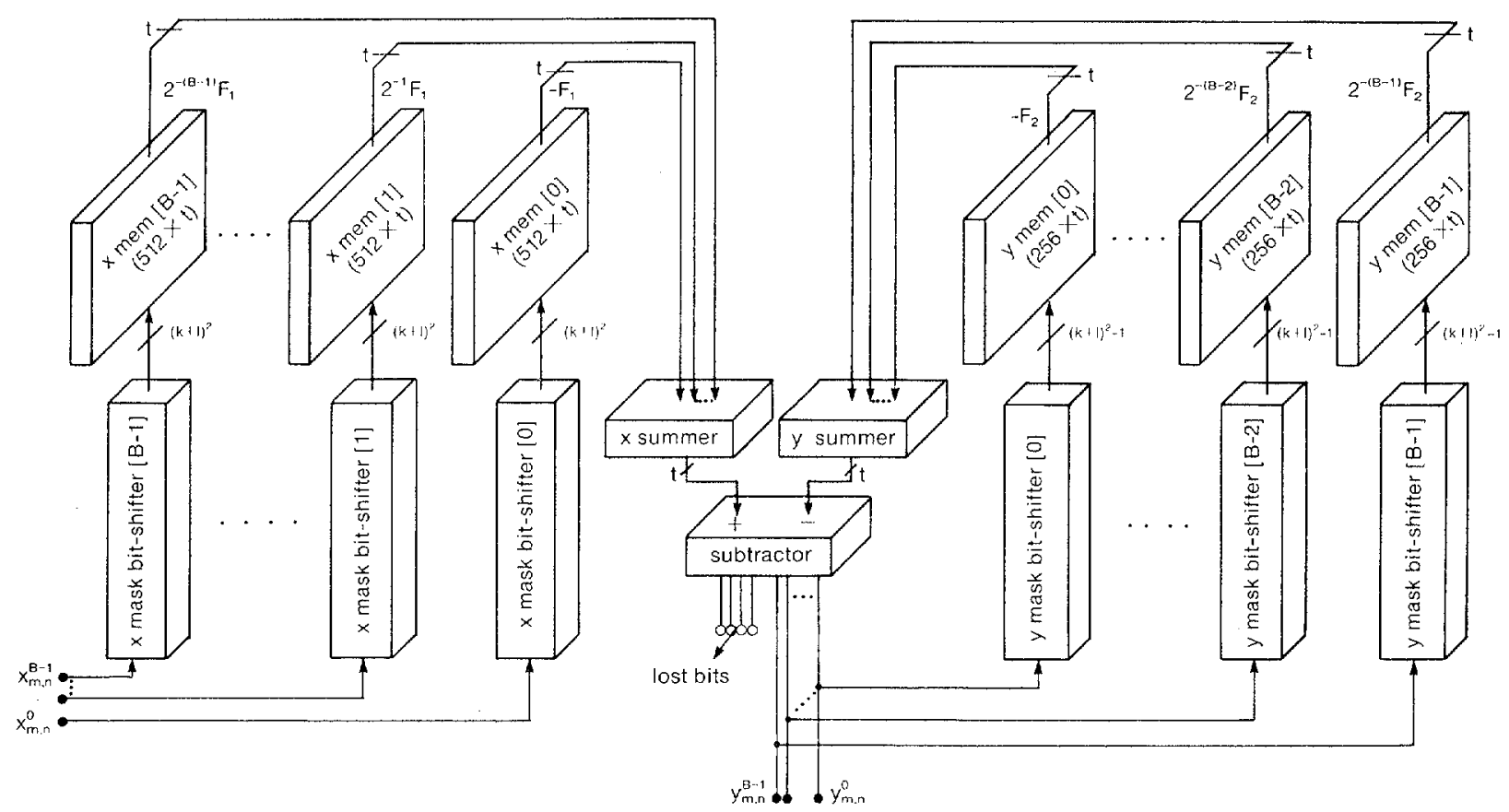

Fig. 2. Schematic block diagram of the distributed arithmetic implementation of second-order section.

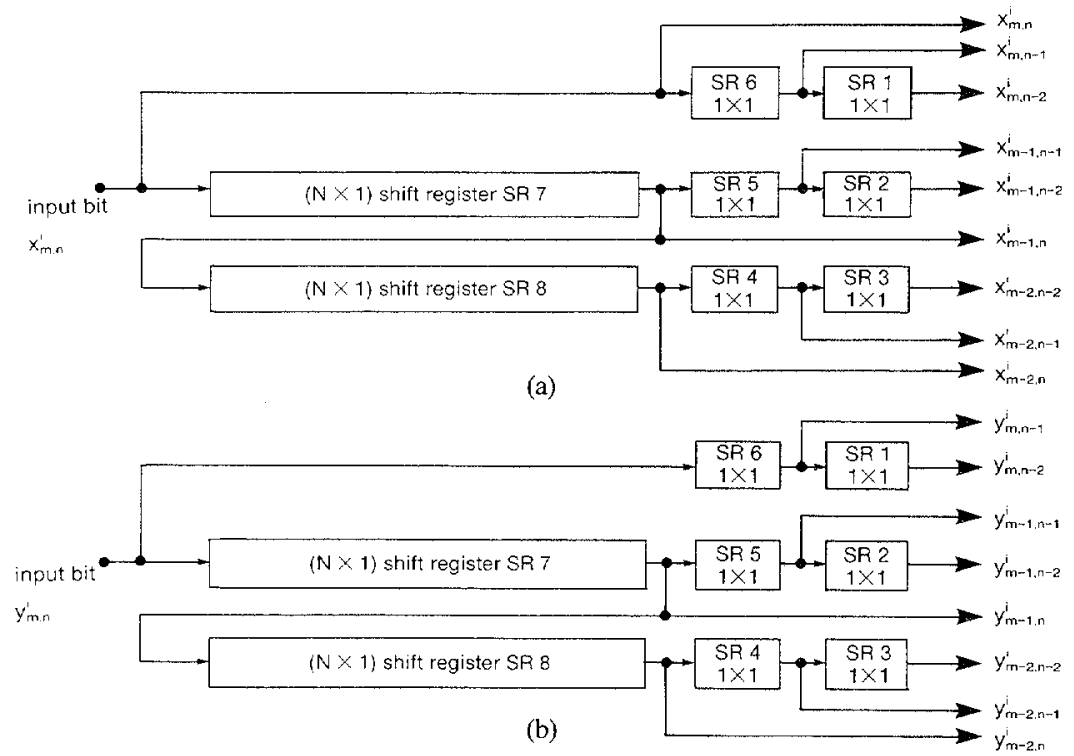

Fig. 3. (a) $X$ mask bit shifter. (b) $Y$ mask bit shifter.

it is possible to write (3) in terms of the two functions $F_{1}$ $(\cdot)$ and $F_{2}(\cdot)$ as

$$
\begin{aligned}
y_{m, n}= & \sum_{s=1}^{B-1} F_{1}^{s}(\cdot) 2^{-s}-F_{1}^{0}(\cdot) \\
& -\sum_{s=1}^{B-1} F_{2}^{s}(\cdot) 2^{-s}+F_{2}^{0}(\cdot)
\end{aligned}
$$

where

$$
\begin{gathered}
F_{1}^{0}\left(x_{m, n}^{0}, x_{m, n-1}^{0}, x_{m, n-2}^{0}, \cdots, x_{m-2, n-2}^{0}\right) \\
=a_{00} x_{m, n}^{0}+a_{01} x_{m, n-1}^{0}+a_{02} x_{m, n-2}^{0} \\
+\cdots+a_{22} x_{m-2, n-2}^{0} .
\end{gathered}
$$

$F_{2}^{0}$ can be expressed in a similar fashion.
The distributed arithmetic realization of (8) consists mainly of four building blocks: 1) mask bit shifters, 2) memories, 3) summers, and 4) subtractor. A schematic block diagram of the implementation is shown in Fig. 2.

The functions $F_{1}^{s}(\cdot)$ and $F_{2}^{s}(\cdot)(8)$ have a finite number of possible outcomes $2^{9}$ and $2^{8}$, respectively. The arguments of the functions $F_{1}^{s}(\cdot)$ and $F_{2}^{s}(\cdot)$ are generated with the $X$ and $Y$ mask bit shifters shown in Fig. 3(a) and (b), respectively. The number of each $X$ and $Y$ mask bit shifters is equal to the word length $B$ in bits. For a secondorder section, the amount of storage required is the previous two columns and two rows of both the input and output arrays. Fig. 4(a) and (b) illustrates the storage allocation in the $X$ mask bit-shifters, and in the $Y$ mask bitshifters, respectively, for the computation of the $(m, n)$ th 


$$
\begin{aligned}
& {\left[x_{m-1, n}^{i}, \ldots, x_{m-1, N}^{i}, x_{N-2,0}^{i}, \ldots, x_{n-2, n+1}^{j}, x_{m-2, n}^{j}\right]+S R \quad 8} \\
& {\left[x_{m, n}^{i}, \ldots, x_{m, N}^{i}, x_{m-1,0}^{i}, \ldots, x_{m-1, n+1}^{i}, x_{m-1, n}^{i}\right]+S R 7} \\
& {\left[x_{n, n-1}^{i}\right]+S R 6} \\
& {\left[\mathrm{x}_{\mathrm{HE}-1, \mathrm{n}-1}^{\mathrm{i}}\right]+\mathrm{SR} 5} \\
& {\left[x_{m-2, n-1}^{i}\right]+\operatorname{SR} 4} \\
& {\left[x_{m-2, n-2}^{\mathrm{i}}\right] \rightarrow \mathrm{SR} 3} \\
& {\left[\mathrm{x}_{\mathrm{m}-1, \mathrm{n}-2}^{\mathrm{i}}\right] \rightarrow \mathrm{SR} 2} \\
& {\left[x_{m, 12-2}^{i}\right] \rightarrow S R \quad 1}
\end{aligned}
$$

(a)

$$
\begin{aligned}
& {\left[y_{n-1, n}^{i}, \ldots, y_{n-1, N}^{i}, y_{n-2,0}^{i}, \ldots, y_{m-2, n+1}^{i}, y_{m-2, n}^{i}\right]+S R 8} \\
& {\left[y_{m, n}^{i}, \ldots, y_{m, N}^{i}, y_{m-1,0}^{i}, \ldots, y_{n-1, n+1}^{i}, y_{m-1, n}^{i}\right]+S R 7} \\
& {\left[y_{m, n-1}^{i}\right]+S R \sigma} \\
& {\left[y_{m-1, n-1}^{i}\right]+S R 5} \\
& {\left[y_{m-2, n-1}^{i}\right]+\operatorname{sR} 4} \\
& {\left[y_{n-2, n-2}^{i}\right]+\operatorname{sR~} 3} \\
& {\left[y_{m-1, n-2}^{\dot{j}}\right]+\operatorname{SR} 2} \\
& {\left[y_{n, n-2}^{j}\right]+S R 2}
\end{aligned}
$$

(b)

Fig. 4. (a) Storage allocation in the $i$ th $X$ mask bit shifter for the computation of the $(m, n)$ th output sample. (b) Storage allocation in the $i$ th $Y$ mask bit shifter for the computation of the $(m, n)$ th output sample.

output sample of an input array $(M \times N)$. The symbol " $\rightarrow$ " is used to indicate that the elements on the left are stored in the shift registers on the right. Shift registers $S R 7$ and $S R 8$ are $N$ bits long and SR1-SR6 are single bit registers.

The memories shown in Fig. 2 are of two sizes, $512 \times$ $t(X \mathrm{MEM})$ and $256 \times t(Y \mathrm{MEM})$, where $t$ represents the coefficient's precision in bits. All linear combinations of the nine coefficients $a_{k}, l$ 's in (3) are stored in $512 \times t$ memories. Similarly, all possible combinations of the eight $b_{i}, j$ 's are stored in the $256 \times t$ memories. In general, the total memory requirement is in the order of $2 B\left(2^{(K+1)^{2}}\right)$ $x t$ where $K$ is the order of the filter.

Each of the two summers shown in Fig. 2 is a tree of adders with $B$ input words. The outputs of the XMEM's $F_{1}^{0}(\cdot)$ to $F_{1}^{B-1}(\cdot)$ are summed in the adder tree of the $X$ summer. The bit shift $2^{-s}$ associated with each function $F_{1}^{s}(\cdot)$ can be either hardwired into the adder's inputs or preprogrammed into the memories. The $Y$ summer has the same configuration as the $X$ summer. For $B$ inputs into a summer there $\operatorname{are} \log _{2} B$ levels of adders in the adder tree and $B-1$ adders are required. When operating on wider words (e.g., $t=16$ ), a carry look-ahead generator can be used together with each adder to achieve higher operating speeds. finally, the subtractor computes the difference between the summations $\Sigma F_{1}^{s}(\cdot) 2^{s}-F_{1}^{0}(\cdot)$ and $\Sigma F_{2}^{s}(\cdot) 2^{s}-$ $F_{2}^{0}(\cdot)$.

\section{ERROR ANALYSIS}

The mean-square error for the distributed arithmetic implementation of 2-D recursive filters is derived in this section. There are basically two different sources of error. The first is due to quantization of the input samples and the second is due to the necessary use of finite word length arithmetic.

In the distributed arithmetic approach, the outputs $y_{m, n}$ are computed from (8) and it is assumed that all computations are performed using infinite word lengths and the output also is of infinite word length representation.

The first place where quantization of the input signal may take place is at the analog-to-digital converter.

Let $\tilde{\mathrm{x}}_{m, n}$ be $x_{m, n}$ quantized (rounded) to $B$ bits including the sign bit and $x_{m, n}^{i}$ be $x_{m, n} i$ th bit. The quantization error is

$$
\epsilon_{m, n}^{\prime}=\left\{\tilde{x}_{m, n}-x_{m, n}, \quad m \geq 0 \text { and } n \geq 0\right\}
$$

with $-2^{-B} \leq \epsilon_{m, n}^{\prime}<2^{-B}$. $\epsilon_{m, n}^{\prime}$ is an uncorrelated sequence with zero mean and a variance of $2^{-2 B} / 3$ [8].

If the input is quantized and all computations are performed with infinite word length, then the output in its infinite word length representation is

$$
v_{m, n}=\sum_{k=0}^{2} \sum_{l=0}^{2} a_{k, l} \tilde{x}_{m-k, n-l}-\sum_{\substack{i=0 \\(i+j) \neq 0}}^{2} \sum_{j=0}^{2} b_{i j} v_{m-i, n-j}
$$

Defining the error at the output of the filter due to quantization of the input as

$$
f_{m, n}=v_{m, n}-y_{m, n} .
$$

Substituting (2), (9), and (10) into (11) gives

$$
f_{m, n}=\sum_{j=0}^{2} \sum_{j=0}^{2} a_{i, j} \epsilon_{m-i, n-j}^{\prime}-\sum_{\substack{i=0 \\(i+j) \neq 0}}^{2} \sum_{j=0}^{2} b_{i, j} f_{m-i, n-j}
$$

The quantization error in (12) is an uncorrelated sequence with zero mean and the variance is given by

$$
\left\langle f_{m, n}^{2}\right\rangle=\left\langle\epsilon_{m, n}^{\prime 2}\right\rangle \sum_{m=0}^{\infty} \sum_{n=0}^{\infty} h_{m, n}^{2}
$$

where $\left\langle\epsilon_{m, n}^{\prime 2}\right\rangle$ is the variance of the input quantization error; and $h_{m, n}$ is the inverse $z$-transform of the filter transfer function $H\left(z_{1}, z_{2}\right)$. Hence

$$
\left\langle f_{m, n}^{2}\right\rangle=\frac{2^{-2 B}}{3} \sum_{m=0}^{\infty} \sum_{n=0}^{\infty} h_{m, n}^{2} .
$$

The output signal $v_{m, n}$ from the infinite length register filter when the inputs are quantized is given by 


$$
\begin{aligned}
v_{m, n}= & \sum_{i=1}^{B-1} 2^{-i} F_{1}^{i}\left(\tilde{x}_{m, n}^{i}, \tilde{x}_{m-1, n}^{i}, \cdots, \tilde{x}_{m-2, n-2}^{i}\right) \\
& -F_{1}^{0}\left(\tilde{x}_{m, n}^{0}, \tilde{x}_{m-1, n}^{0}, \cdots, \tilde{x}_{m-2, n-2}^{0}\right) \\
& -\sum_{i=1}^{B-1} 2^{-i} F_{2}^{i}\left(v_{m-1, n}^{i}, v_{m-2, n}^{i}, \cdots, v_{m-2, n-2}^{i}\right) \\
& +F_{2}^{0}\left(v_{m-1, n}^{0}, v_{m-2, n}^{0}, \cdots, v_{m-2, n-2}^{0}\right) .
\end{aligned}
$$

The rounded values of $F_{1}(\cdot)$ and $F_{2}(\cdot)$, due to finite word length of the memories, are defined as

$$
\begin{aligned}
& \tilde{F}_{\mathrm{I}}^{i}\left(\tilde{x}_{m, n}^{i}, \tilde{x}_{m-1, n}^{i}, \cdots, \tilde{x}_{m-2, n-2}^{i}\right) \\
& \quad=F_{1}^{i}\left(\tilde{x}_{m, n}^{i}, \tilde{x}_{m-1, n}^{i}, \cdots, \tilde{x}_{m-2, n-2}^{i}\right)+\eta_{m, n}^{i}
\end{aligned}
$$

and

$$
\begin{aligned}
& \tilde{F}_{2}^{i}\left(v_{m-1, n}^{i}, v_{m-2, n}^{i}, \cdots, v_{m-2, n-2}^{i}\right) \\
& =F_{2}^{i}\left(v_{m-1, n}^{i}, v_{m-2, n}^{i}, \cdots, v_{m-2, n-2}^{i}\right)+\gamma_{m, n}^{i}
\end{aligned}
$$

where $\eta_{m, n}^{i}$ and $\gamma_{m, n}^{i}$ are due to roundoff. The $\eta_{m, n}^{i}$ 's are zero mean, uncorrelated, and each have a variance $2^{-2 t} / 3$, where $t$ is the word length in bits chosen to represent the precomputed sum of partial products. Similarly, $\gamma_{m, n}^{i}$ are zero mean, uncorrelated, and each have variance $2^{-2 t} / 3$.

The rounded output can be represented as

$$
\begin{aligned}
\omega_{m, n}= & \sum_{i=1}^{B-1}\left[2^{-i} \tilde{F}_{1}^{i}\left(\tilde{x}_{m, n}^{i}, \tilde{x}_{m-1, n}^{i}, \cdots, \tilde{x}_{m-2, n-2}^{i}\right)+\epsilon_{q 1}^{i}\right] \\
& -\tilde{F}_{1}^{0}\left(\tilde{x}_{m, n}^{0}, \tilde{x}_{m-1, n}^{0}, \cdots, \tilde{x}_{m-2, n-2}^{0}\right) \\
& -\sum_{i=1}^{B-1}\left[2^{-i} \tilde{F}_{2}^{i}\left(v_{m-1, n}^{i}, v_{m-2, n}^{i}, \cdots, v_{m-2, n-2}^{i}\right)\right. \\
& \left.\quad+\epsilon_{q 2}^{i}\right] \\
& +F_{2}^{0}\left(v_{m-1, n}^{0}, v_{m-2, n}^{0}, \cdots, v_{m-2, n-2}^{0}\right)+\epsilon_{q}^{\prime}
\end{aligned}
$$

where

$\epsilon_{q 1}^{i}$ and $\epsilon_{q 2}^{i}$ are the quantization errors when $\tilde{F}_{1}^{i}(\cdot) 2^{-i}$ and $\tilde{F}_{2}^{i}(\cdot) 2^{-i}$ are rounded from $(t+i)$ bits to $t$ bits with variances $2^{-2 t} / 3$

and

$\epsilon_{q}^{\prime}$ is the quantization error when the result $\omega_{m, n}$ is truncated from $t$ bits to $B$ bits with variance $2^{-2 B} / 3$.

Let $g_{m, n}$ be the accumulated truncation error at the output of the filter defined as

$$
\begin{aligned}
g_{m, n} & =v_{m, n}-\omega_{m, n} \\
& =\delta_{m, n}-\sum_{\substack{i=0 \\
i+j \neq 0}}^{2} \sum_{\substack{j=0 \\
i \neq j}}^{2} b_{i j} g_{m-i, n-j}
\end{aligned}
$$

where

$$
\begin{aligned}
\delta_{m, n}= & -\sum_{i=1}^{B-1}\left(\eta_{m, n}^{i} 2^{-i}+\epsilon_{q 1}^{i}\right)+\eta_{m, n}^{0} \\
& +\sum_{i=1}^{B-1}\left(\gamma_{m, n}^{i} 2^{-i}+\epsilon_{q 2}^{i}\right)-\gamma_{m, n}^{0}-\epsilon_{q}^{\prime} .
\end{aligned}
$$

The variance of $g_{m, n}$ can be calculated as in [9]

$$
\begin{aligned}
\left\langle g_{m, n}^{2}\right\rangle= & \sum_{m=0}^{\infty} \sum_{n=0}^{\infty} d_{m, n}^{2}\left\{E \left[\sum_{i=1}^{B-1}\left(\eta_{m, n}^{i} 2^{-i}+\epsilon_{q 1}^{i}\right)-\eta_{m, n}^{0}\right.\right. \\
& \left.-\sum_{i=1}^{B-1}\left(\gamma_{m, n}^{i} 2^{-i}+\epsilon_{q 2}^{i}\right)+\gamma_{m, n}^{0}+\epsilon_{q}^{\prime}\right]^{2} \\
& -E^{2}\left[-\sum_{i=1}^{B-1}\left(\eta_{m, n}^{i} 2^{-i}+\epsilon_{q 1}^{i}\right)+\eta_{m, n}^{0}\right. \\
& \left.\left.+\sum_{i=1}^{B-1}\left(\gamma_{m, n}^{i} 2^{-i}+\epsilon_{q 2}^{i}\right)-\gamma_{m, n}^{0}-\epsilon_{q}^{\prime}\right]\right\}
\end{aligned}
$$

where $d_{m, n}^{2}$ is the inverse $z$-transform of $1 / D\left(z_{1}, z_{2}\right)$, where $D\left(z_{1}, z_{2}\right)$ is the denominator of the transfer function $H\left(z_{1}\right.$, $\left.z_{2}\right)$.

Since all errors are uncorrelated, (14) becomes

$$
\begin{aligned}
\left\langle g_{m, n}^{2}\right\rangle= & \sum_{m=0}^{\infty} \sum_{n=0}^{\infty} d_{m, n}^{2}\left\{\sum_{i=1}^{B-1}\left(\left\langle\eta_{m, n}^{i^{2}}\right\rangle 2^{-2 i}+\left\langle\epsilon_{q 1}^{i^{2}}\right\rangle\right)\right. \\
& +\left\langle\eta_{m, n}^{0^{2}}\right\rangle+\sum_{i=1}^{B-1}\left(\left\langle\gamma_{m, n}^{i^{2}}\right\rangle 2^{-2 i}+\left\langle\epsilon_{q 2}^{i 2}\right\rangle\right) \\
& \left.+\left\langle\gamma_{m, n}^{0^{2}}\right\rangle+\left\langle\epsilon_{q}^{\prime 2}\right\rangle\right\} \\
= & \sum_{m=0}^{\infty} \sum_{n=0}^{\infty} d_{m, n}^{2} \\
& \cdot\left\{\frac{2^{-2 t}}{3}\left[\frac{2\left(2^{-2}-2^{-2 B}\right)}{1-2^{-2}}+2 B\right]+\frac{2^{-2 B}}{3}\right\} .
\end{aligned}
$$

Then the output error has a zero mean and a variance

$$
\begin{aligned}
\left\langle\epsilon_{m, n}^{2}\right\rangle= & \left\langle f_{m, n}^{2}\right\rangle+\left\langle g_{m, n}^{2}\right\rangle \\
= & \frac{2^{-2 B}}{3}\left\{\sum_{m=0}^{\infty} \sum_{n=0}^{\infty} h_{m, n}^{2}+\sum_{m=0}^{\infty} \sum_{n=0}^{\infty} d_{m, n}^{2}\right\} \\
& +\frac{2^{-2 t}}{3}\left[\frac{2\left(2^{-2}-2^{-2 B}\right)}{1-2^{-2}}+2 B\right] \sum_{m=0}^{\infty} \sum_{n=0}^{\infty} d_{m, n}^{2} .
\end{aligned}
$$

Equation (15) can also be used to determine $B$ and $t$ for a specified signal-to-noise ratio.

Example: For the high emphasis filter described in [1] (which will be used to demonstrate the distributed arithmetic implementation in Section V)

$$
\begin{aligned}
& \sum_{m=0}^{\infty} \sum_{n=0}^{\infty} h_{m, n}^{2} \simeq 0.823602923 \text { and } \\
& \sum_{m=0}^{\infty} \sum_{n=0}^{\infty} d_{m, n}^{2} \simeq 1.12468584
\end{aligned}
$$

with $B=8$ and $t=16$, the output mean-squared error

$$
\left\langle\epsilon_{m, n}^{2}\right\rangle \simeq 9.91096 \times 10^{-6} \text {. }
$$

\section{Real-Time Image Processing}

The need for real-time digital processing became evident with the increasing utilization of television imaging 


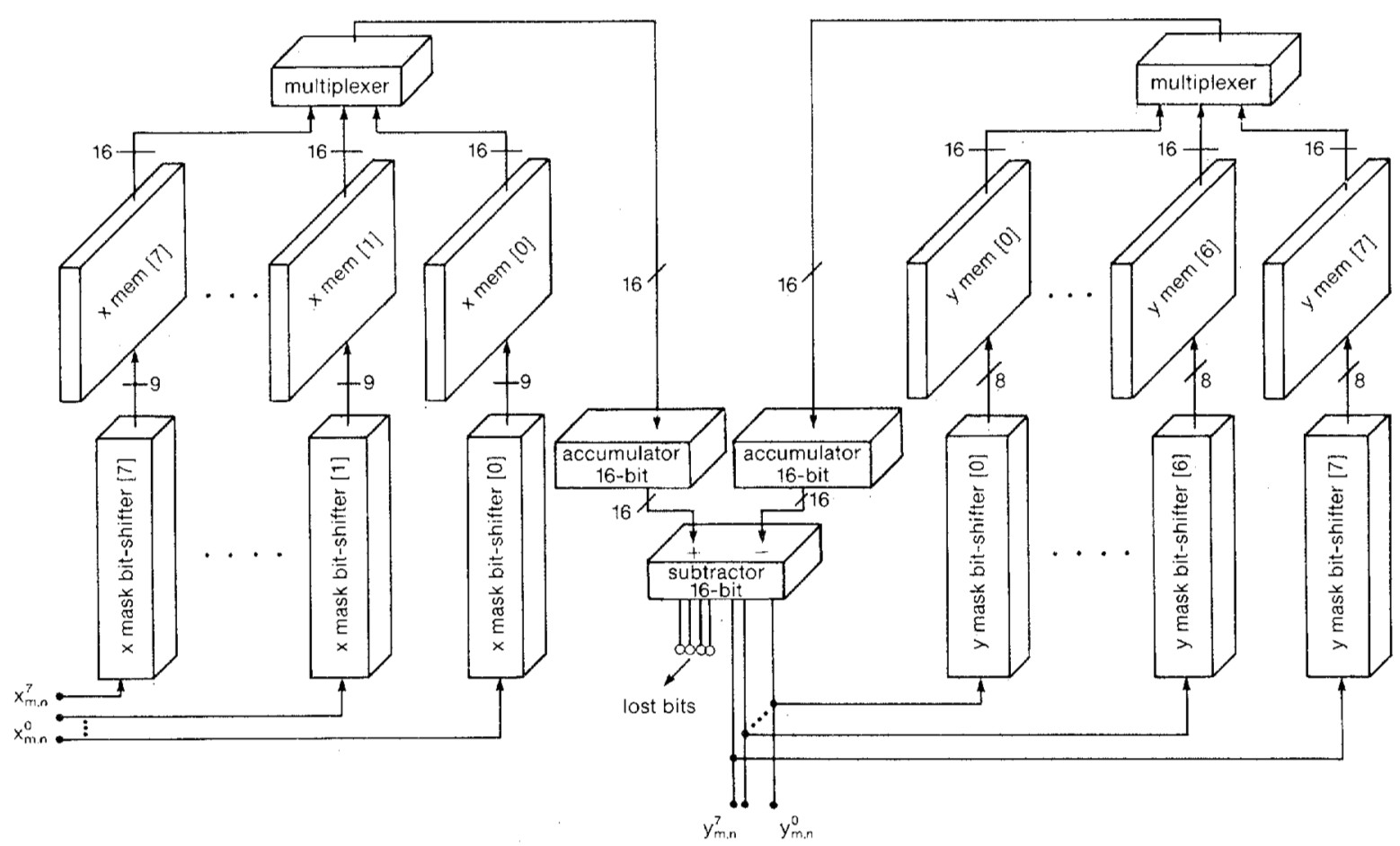

Fig. 5. Schematic block diagram of a distributed implementation with serial additions.

to the industrial, medical, and military environments. The term "real-time image processing" can be defined as "the processing of images at a speed such that the data rate of the processed images is the same as the input one." If we consider an image size of $M \times N$ pixels and a TV scan rate of $L$ images per second, the input data rate,

$$
R=M \times N \times L \text { pixels } / \mathrm{s} .
$$

In order to have the same output data rate, all the operations that are required to produce an output pixels have to be done within $1 / R \mathrm{~s}$. Hence, the quantity $1 / R$ can be viewed as the period of the clock that controls the image processing system. With a display size of $256 \times 256$ pixels and a TV scan rate of 30 images per second, the data throughput rate $R=256^{2} \times 30=1.966 \times 10^{6} \mathrm{pixels} / \mathrm{s}$ or one pixel every $508.6 \mathrm{~ns}$.

Real-time digital image processing requires a large amount of number crunching to be performed at high speed. Conventional computer architectures which operate in a sequential manner are $\mathrm{I} / \mathrm{O}$ bound when performing such tasks. The resulting computational problems are attacked by combination of fast processors and advanced system architectures. Even with these systems, most of the image processing algorithms can only be done near real-time. The real-time processing algorithms are limited to pixel manipulations and include such operations as wrapping, rotation, zooming, and small image convolutions. This section demonstrates that distributed arithmetic implementation of 2-D recursive second-order filters can perform real-time spatial filtering of image $256 \times 256$ pixels.

Consider the implementation shown in Fig. 2 for an image of $256 \times 256$ pixels. The coefficients are quantized to 16 bits. All computations are done with $t=16$ bits of precision and the input and output signals are represented by $B=8$ bits. The mask bit-shifters each consist of two $(256 \times 1)$ shift registers and 6 single bit shift registers. These can be configured from MOS AM2856 [11], dual $(256 \times 1)$ shift registers capable of $2.5 \mathrm{MHz}$ shift cycle, and TTL 74S174s, hex D-type flip-flops. The memories, if constructed from AM27S191 $(2 \mathrm{~K} \times 8)$ PROM's, 32 packages are required for the XMEMs and YMEMs. The summers and subtractor can be configured from TTL 74S181s and TTL 74S182s.

The package count for the distributed arithmetic implementation is 139 IC's and the power consumption is $36 \mathrm{~W}$. The estimated cost is approximately $\$ 870(\text { U.S. })^{2}$. The inherent parallelism of the architecture reduces the data rate to a memory fetch, $\left(\log _{2} B\right)+1$ additions and register's delay (due to recursive part). Using standard available TTL integrated circuits and bipolar memory, the time for a 16 bit addition is $19 \mathrm{~ns}$ and that of a memory access is $50 \mathrm{~ns}$ [12], [13]. In addition, the minimum set up time and maximum propagation delay of the shift registers are $100 \mathrm{~ns}$ and $280 \mathrm{~ns}$, respectively [11]. This gives a maximum cycle time of $(4 \times 19)+50+380=506 \mathrm{~ns}$ or a minimum data rate of $1.98 \mathrm{MHz}$. This data rate is within what is required for real-time filtering. Higher data rate (e.g., 6.4 $\mathrm{MHz}$ ) can be attained if faster shift registers such as the TRW TDC1006J with a maximum propagation delay of $30 \mathrm{~ns}$ are used. However, with this replacement, the total cost is approximately doubled. If the memories outputs are shifted and summed serially instead of in parallel, realtime filtering can be achieved if faster shift registers such as the TRW TDC1006J are used. Fig. 5 shows the schematic block diagram of the distributed arithmetic realiza-

\footnotetext{
${ }^{2}$ Cost refers to prices in 1984 in U.S. dollars.
} 


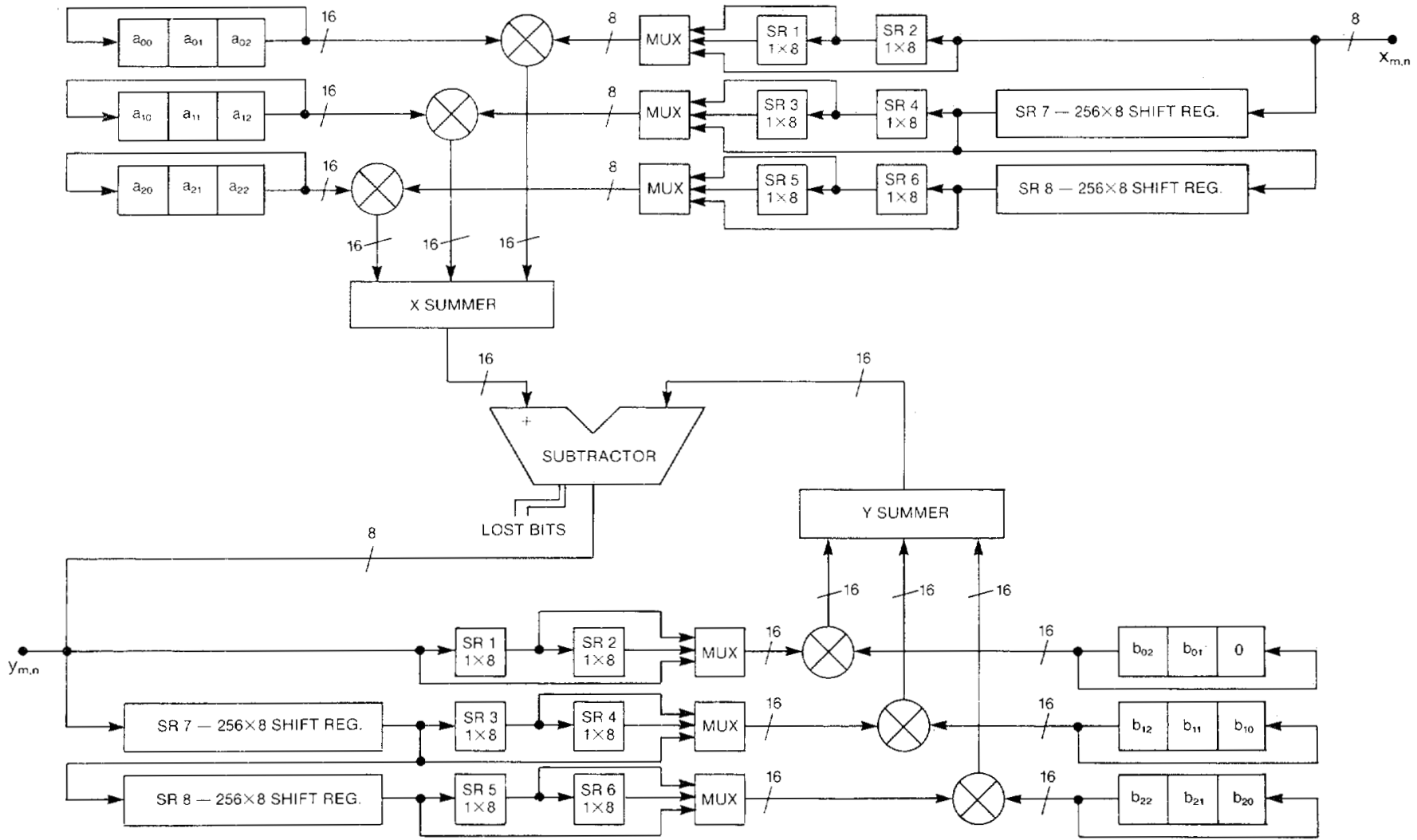

Fig. 6. Multiplier filter functional block diagram.

\begin{tabular}{|c|c|c|c|c|}
\hline $\begin{array}{l}\text { Timplenentation } \\
\text { Seheme }\end{array}$ & Application & $\begin{array}{c}\text { Throughpet } \\
\text { Rate }\end{array}$ & No. of IC & $\begin{array}{l}\text { Estimated Cost } \\
\text { (US } \$)^{*}\end{array}$ \\
\hline $\begin{array}{l}\text { Distributed } \\
\text { Arithmetic } \\
\text { with paralled } \\
\text { addition }\end{array}$ & real-time & $\begin{array}{l}1.98 \mathrm{MHz} \\
6.4 \mathrm{MHz}\end{array}$ & $\begin{array}{l}139 \\
155\end{array}$ & $\begin{array}{l}\$ 870 \\
\$ 1770\end{array}$ \\
\hline $\begin{array}{l}\text { Distributed } \\
\text { Arithinetic } \\
\text { with serial } \\
\text { addition }\end{array}$ & rea1-time & $4 \mathrm{MHz}$ & 105 & $\$ 1550$ \\
\hline $\begin{array}{l}\text { Distributed } \\
\text { Arithmetic } \\
\text { with serial } \\
\text { addition } \\
\text { (hardware } \\
\text { built) }\end{array}$ & $\begin{array}{l}\text { non } \\
\text { rea1-time }\end{array}$ & $880 \mathrm{KHz}$ & 89 & $\$ 350$ \\
\hline Multiplier & $\begin{array}{l}\text { non } \\
\text { real-time }\end{array}$ & $1.17 \mathrm{MHz}$ & 67 & $\$ 2200$ \\
\hline
\end{tabular}

Fig. 7. Comparison among the various implementation schemes for the processing of $256 \times 256$ pixel images.

tion with serial additions. In this case, the data rate is $1 /$ $[(9 \times 19)+50+30]=4 \mathrm{MHz}$.

The memories in Fig. 2 can be replaced by a number of multipliers operating in parallel. Fig. 6 shows the block schematic of a multiplier implementation of a 2-D recursive filter. A minimum of six multipliers are needed, three each for the input and output masks, respectively. Using LSI (large scale integration) $16 \mathrm{bit} / \mathrm{multiplier/accumulator}$ TRW TDC1010J having a speed of $115 \mathrm{~ns}$ and the same adders and shift registers as before, the cycle time will be $115 \times 3+(19 \times 6)+19+280+100=858$ ns. Hence real-time filtering cannot be achieved via the multiplier implementation. The package count in this case is $67 \mathrm{IC}$ 's, power consumption is $42 \mathrm{~W}$, and the cost is approximately $\$ 2200$ (U.S.). Images larger than $512 \times 512$ pixels require the use of esoteric logic types. With such logic families, the penalty for state-of-the-art performance is increased cost. The above discussions are summarized in the table of Fig. 7 for real-time and nonreal-time applications. ${ }^{3}$

\section{HARDWARE DESCRIPTION}

A prototype of the distributed arithmetic implementation of a $2-D$ recursive filter was built using mainly TTL

${ }^{3}$ Some of the preliminary work on the implementation of a 2-D digital filter through distributed arithmetic can be found in [14] and [15]. 


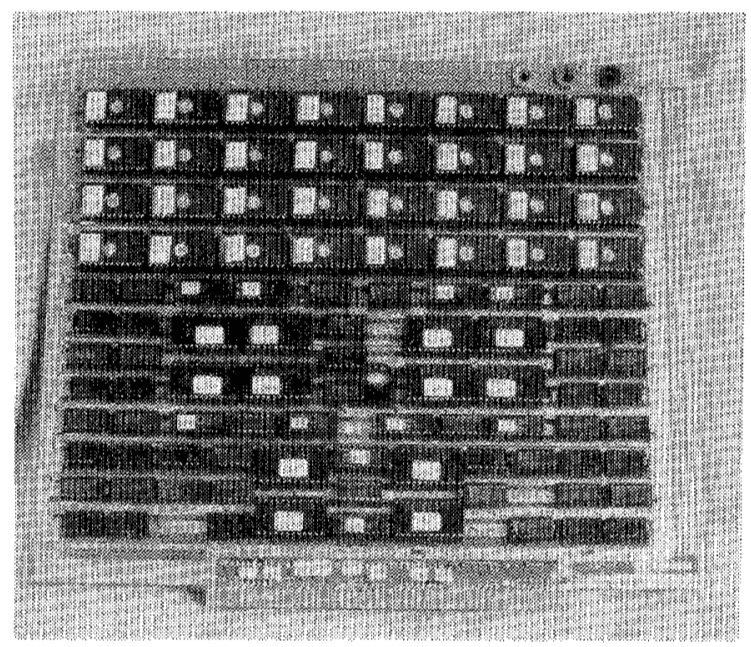

Fig. 8. Photograph of the 2-D second-order distributed filter.

components. In this prototype, serial additions rather than parallel conditions were used in order to reduce the hardware size. The prototype assumes that the input and output samples are 8 bits in length, while all the intermediate computations are done in 16 bit precision.

The $X$ and $Y$ mask bit shifters are constructed using TTL 74S174 hex D-type flip-flops and MOS AM 2856 dual $(256 \times 1)$ static shift registers. The memories are configurated from $2716(2 \mathrm{~K} \times 8)$ EPROM's (erasable programmable read-only memory). The use of EPROM's yields a more flexible filter at the expense of a slower throughput rate. With serial addition, an accumulator type circuit is required. This was constructed using four $74 L S 1814$ bit ALU's, one 74LS182 carry look-ahead generator, and two TTL octal latches. The subtractor has the same configuration. A schematic block diagram of this approach is shown in Fig. 5. Also in this hardware, the bit shifts $2^{-s}$ associated with each function $F^{s}(\cdot)$ were preprogrammed into the memories, which implies that the memories are not identical.

The operational sequence for this implementation is now outlined.

Assume that input image to be filtered is of size $M \times$ $N$ and that we are calculating the first output sample for the $m$ th row. For simplicity assume all initial conditions are zero. This means that registers $S R 1-S R 8$ of all the input and output mask bit-shifters are cleared and the subtractor output is zeroed. With the aid of Fig. 5, the steps involved in the computation of $y_{m, o}$ are as follows.

Step 1: Each input bit $x_{m, o}^{i}$ is shifted into the corresponding $i$ th mask bit-shifter, and simultaneously, the subtractor's output bits are shifted in a similar fashion into the $Y$ mask bit-shifters.

Step 2: The binary vector output of each of the $X$ mask bit-shifters address one of the $B X$ MEM's. Similarly, the $Y$ MEM's are addressed by the $Y$ mask bit-shifters outputs.

Step 3: The fetched memory contents of XMEM's are added in the $X$ accumulator with a sign change for the contents of the XMEM representing the function $F_{1}^{0}(\cdot)$.
Similarly, the fetched memory contents of the YMEM's are added in the $Y$ accumulator.

Step 4: The difference in the outputs of the $X$ and $Y$ accumulators is computed in the subtractor. The subtractor now contains $y_{m, o}$.

Step 5: All the registers in the $X$ and $Y$ mask bitshifters are strobed so that all the delay versions of the input and output samples are available at the outputs of the $X$ and $Y$ mask bit-shifters.

To calculate the next output sample, steps 1 to 5 are repeated with $x_{m, l}$. Continuing along the $m$ th row after $N$ samples, the entire row will have been processed. For the next row of computation registers SR1-SR6 of the $X$ and $Y$ mask bit-shifters are cleared so that the proper initial condition is retained. The steps are repeated in a similar fashion until the last element $y_{M, N}$ is computed.

In this serial implementation, the memory is enabled in sequence for serial addition as opposed to the parallel implementation, where all memories are enabled simultaneously. This was accomplished by using a simple counterdecoder circuit. The counter is used to keep track of the number of additions as well as to strobe the latches of the subtractor. The counter also determines when to disable the clock to the accumulator and subtractor latches upon the required number of arithmetic operation is complete. This prototype costs approximately $\$ 350$ (U.S.) and can operate up to $880 \mathrm{kHz}$ for an image size up to $256 \times 256$ pixels. A photograph of the prototype is shown in Fig. 8.

The performance of the distributed arithmetic implementation is demonstrated using a second-order IIR filter drawn from [1]. This filter has a magnitude response that is generally high pass and a linear phase characteristic over most of the frequency domain. The filter has been designed to have a gain equal to 1 beyond the radial frequency of $0.7 \pi$ and 0.1 below the radial frequency of $0.3 \pi$. The transition band is Gaussian between $0.3 \pi$ and $0.7 \pi$. The contour plot of this filter is shown in Fig. 9. Two different sizes of images are processed with this filter. They are the $128 \times 128$ pixels crest image and the $128 \times 256$ boat image shown in Fig. 10(a) and 11(a), respectively. 


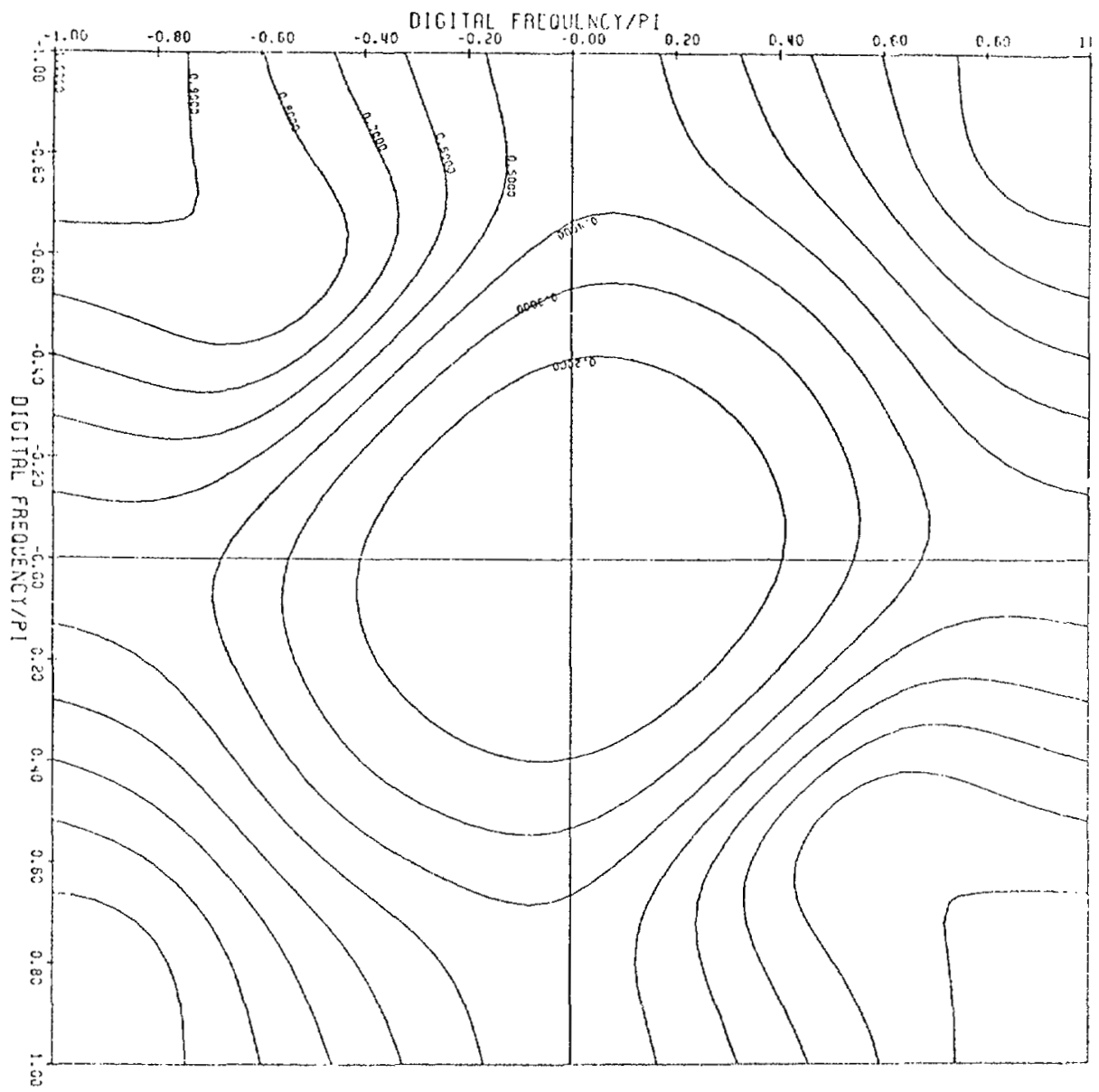

Fig. 9. Contour plot of the high emphasis filter.

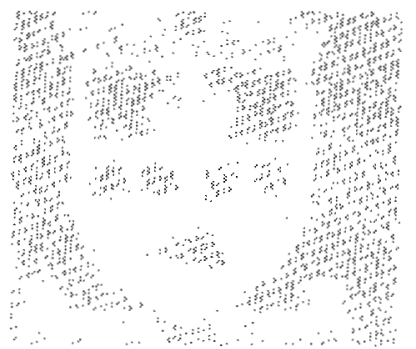

(a)

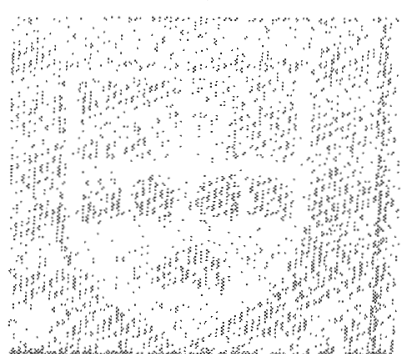

(b)

Fig. 10. (a) Original crest image. (b) Crest image filtered by the high emphasis filter.

The filtered images are shown in Figs. 10(b) and 11(b), respectively. The results agree with the fact that the high emphasis filter passes low-frequency components unchanged and amplifies high-frequency components. Thus,

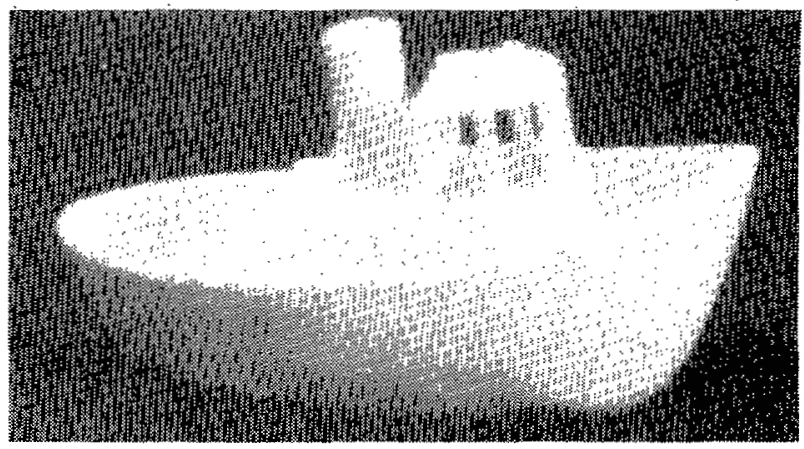

(a)

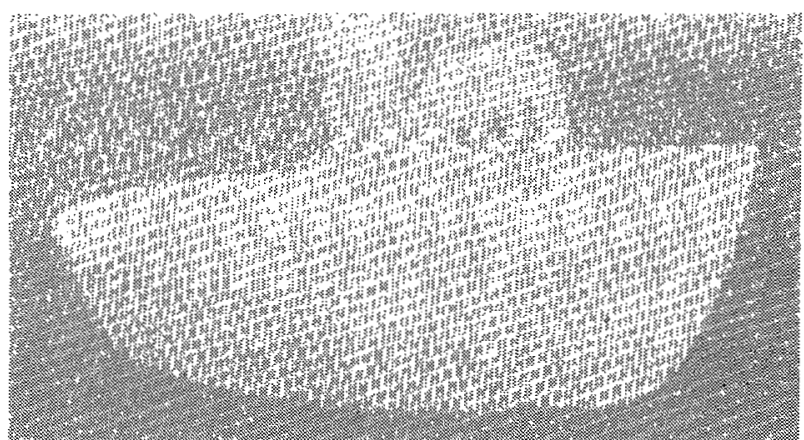

(b)

Fig. 11. (a) Original boat image. (b) Boat image filtered by the high emphasis filter. 
it is demonstrated that the filter has been successfully implemented using the distributed arithmetic structure.

\section{Conclusions}

The distributed arithmetic realization of a second-order recursive digital filter has been presented. The implementation was based on memory fetch, bit-shifts, and additions. This is different from the conventional direct method of filter implementation that uses a number of multipliers operating in parallel for the same throughout. The proposed structure, when compared to the conventional method of filtering, offers significant reductions in cost and power consumption, and can operate in real-time on images as large as $256 \times 256$ pixels. For higher order filters, a split-address technique can be used to reduce the memory size while trading off with some extra additions. Finally, the inherent parallelism in the architecture and the fast growing VLSI device technology with its ability to boost parallel processing suggest that the filters can be economically implemented.

\section{REFERENCES}

[1] E. L. Hall, "A comparison of computations for spatial frequency filtering," Proc. IEEE, vol. 60, no. 7, July 1972.

[2] A. Chottera and G. A. Jullien, "Recursive digital filters in image processing," in Proc. IEEE Int. Conf. ASSP, Apr. 1978, pp. 757-760.

[3] A. Peled and B. Liu, "A new hardware realization of digital filters," IEEE Trans. ASSP, vol. ASSP-22, pp. 456-462, Dec. 1974.

[4] F. J. Taylor, "A distributed arithmetic MFIR filter," IEEE Trans. ASSP, vol. ASSP-32, no. 1, pp. 186, Feb. 1984

[5] J. W. Modestino and R. W. Fries, "Edge detection in noisy images using recursive digital filtering," Computer Graphics and Image Processing, vol. 6, no. 5, Oct. 1977.

[6] A. Chottera and G. A. Jullien, "Designing near linear phase recursive filter using linear programming," in Proc. IEEE Int. Conf. ASSP, May 1977, pp. 88-92.

[7] B. T. O'Connor and T. S. Huang, "Stability of general two-dimensional recursive digital filters," IEEE Trans. ASSP, vol. ASSP-26, Dec. 1978.

[8] B. Liu, "Effects of finite word length on the accuracy of digital filters," IEEE Trans. Circuit Theory, vol. 18, pp. 670-677, Nov. 1971.

[9] A. N. Venetsanopoulos, B. G. Mertzios, and S. H. Mneney, "Effects of finite precision in two-dimensional recursive digital filters," Int. $J$. Electronics, vol. 58, no. 1, pp. 159-174, 1985

[10] M. Ni and J. K. Aggarwal, "Two-dimensional digital filtering and its error analysis," IEEE Trans. Comput., vol. C-23, pp. 942-954, Sept. 1974.

[11] Advanced Micro Devices, Inc., MOS/LSI Data Book, Sunnyvale, CA. 1980.

[12] Texas Instruments, Inc., The TTL Data Book for Design Engineers, 2nd ed., Dallas, TX, 1981.

[13] Signetics Corporation, Signetics Bipolar and MOS Memory Data Manual, Sunnyvale, CA, 1978.

[14] H. Jaggernauth and A. N. Venetsanopoulos, "Distributed arithmetic implementations of two-dimensional filters," in Proc. IEEE Canadian Communications Energy Conf., Montreal, Canada, Oct. 13-15, 1982, pp. $407-410$.

[15] — "Real-time image processing through distributed arithmetic," in Proc. IEEE Int. Symp. Circuits Syst., Newport Beach, CA, May 1983, vol. 1 , pp. 394-397.

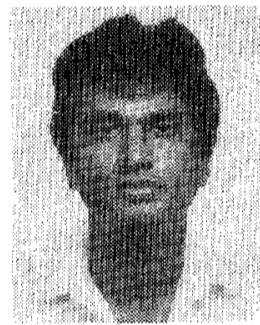

Jeff Jaggernauth was born in Georgetown, Guy ana, in January 1956 . He received the B.A.Sc. degree in electrical engineering from the University of Windsor, Ont., Canada, in 1979 and M.Eng. degree in electrical engineering from the University of Toronto, Ont., Canada, in 1981.

Since 1981 he has worked in the area of telecommunications doing hardware and software designs. He is currently with Siemens Communications Systems, Boca Raton, FL. His interests include real-time image processing and data communications.

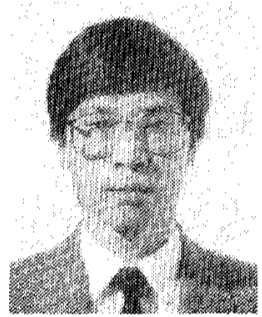

Alexander C. P. Loui was born in Trinidad \& Tobago on February 20, 1961. He received the B.A.Sc. degree in electrical engineering from the University of Toronto, Ont., Canada, in 1983. He is now completing the M.A.Sc. degree, also from the same university.

In the summer of 1982, he worked at Atomic Energy of Canada, Ltd., as a Research Assistant. During the period of 1983-1985, he was working as a Teaching Assistant with the Department of Electrical Engineering, University of Toronto, Ont., Canada. In 1983 he was awarded the NSERC Postgraduate Scholarship from the Natural Sciences and Engineering Research Council of Canada. His research interests include digital signal processing, image processing, and digital communications.

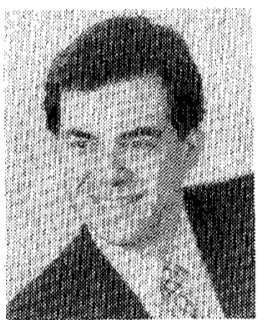

Anastasios N. Venetsanopoulos (S'66-M'69SM'79) received the mechanical and electrical engineering degree from the National Technical University of Athens, Greece, in 1965. He received the M.S., M.Phil., and Ph.D. degrees from the Department of Engineering and Applied Science, Yale University, New Haven, CT, in 1966, 1968, and 1969 , respectively.

He joined the University of Toronto, Canada, in September 1968, where he is now Professor and Chairman of the Communications Group, Department of Electrical Engineering. He was Program Chairman of the International Communications Conference (ICC'78) and will be again for ICC' 86 . He has served as Chairman of the Central Canada Council of IEEE and the Editor of the Canadian Electrical Engineering Journal. He is presently a Fellow of the Engineering Institute of Canada, Associate Editor for Digital Signal Processing of the IEEE TRAnsaCTIONS ON Circuts AND SYsTEMS, and the President of the Canadian Society for Electrical Engineering. His research interests span the fields of digital signal/image processing and digital communications. He has conducted research, taught, and consulted extensively in Europe and North and South America.

Dr. Venetsanopoulos is a member of the Association of Professional Engineers of Ontario, the New York Academy of Sciences, Sigma Xi and the Technical Chamber of Greece. 